\title{
Two-dimensional few cycle optical pulses inside a carbon nanotubes photonic crystal
}

\author{
Yulia V. Dvuzhilova, Ilya S. Dvuzhilov", and Mikhail B. Belonenko \\ Volgograd State University, 400062 Volgograd, Russia
}

\begin{abstract}
We have considered the problem of evolution of the twodimensional few cycle optical pulses (FCOP) inside a photonic crystal made of carbon nanotubes. It has been shown that pulse propagation is stable inside the considered environment and it is possible to control and to change the velocity of the pulse propagation by photonic crystal parameters changing.
\end{abstract}

Nowadays among the popular phenomena of nonlinear and coherent optics the study interest of the dynamic of optical pulses which have a small number of electromagnetic field period oscillations (few cycle optical pulses) is really high. Such pulses are also called light bullets [1]. One of the main characteristics of FCOP is that impossible to split the shape of the electromagnetic pulse into the envelope and the bearing part, this is could be interested for the researches in many ways. That is why in this case we cannot use the multiscale expansion method for solving Maxwell's equations. There is a need to solve Maxwell's equations without discarding any derivatives $[2,3]$. We have to point to the fact that if the medium can be characterized by spatial dispersion then nonlinearity must be taken into account even with the one-dimensional

In this paper we have studied $\pi$-electrons of CNTs using the tight-bond approximation. The law of dispersion for semi-conductor CNTs of zig-zag type can be written as:

$$
E(\boldsymbol{p})= \pm \gamma \sqrt{1+4 \cos \left(a p_{z}\right) \cos (\pi s / m)+4 \cos ^{2}(\pi s / m)}
$$

where $\gamma \approx 2.7 \mathrm{eV}, a=3 b / 2 \hbar, b=0.152 \mathrm{~nm}$ - the distance between neighbours carbon atoms, and the quasi-momentum $\mathbf{p}$ is given as $\left(p_{z}, s\right), s=1,2 \ldots m$.

Finally, an effective equation can be represented as:

$$
\begin{gathered}
\frac{\partial^{2} A_{z}}{\partial x^{2}}+\frac{\partial^{2} A_{z}}{\partial y^{2}}-\frac{n^{2}(x, y)}{c^{2}} \frac{\partial^{2} A_{z}}{\partial t^{2}}+\frac{q}{\pi \hbar} \sum_{m} c_{m} \sin \left(\frac{m a q}{c} A_{z}(t)\right)=0 \\
c_{m}=\sum_{m} a_{m s} b_{m s}, b_{m s}=\int_{-q_{0}}^{q_{0}} d p_{z} \cos \left(\operatorname{map}_{z}\right) F_{0}(\boldsymbol{p})
\end{gathered}
$$

\footnotetext{
* Corresponding author: dvuzhilov.ilya@ volsu.ru
} 
Since, as a result of a numerical solution, the coefficients value $c_{m}$ decrease, we can restrict ourselves to the first few terms of expansion (for our calculations, we have used the first 10)

The initial condition of the potential-vector can be written as:

$$
\begin{array}{r}
A_{t=0}=A_{0} \exp \left\{-\frac{x^{2}}{\gamma^{2}}\right\} \exp \left\{-\left(y-y_{c}\right)^{2} / \beta^{2}\right\} \\
\left.\frac{d A}{d t}\right|_{t=0}=\frac{2 v x}{\gamma^{2}} A_{0} \exp \left\{-\frac{x^{2}}{\gamma^{2}}\right\} \exp \left\{-\left(y-y_{c}\right)^{2} / \beta^{2}\right\}
\end{array}
$$

where $y_{c}$ - the initial position of the pulse about the ordinate axis, $\beta, \gamma-$ are the parameters of the pulse width along the $y$ and $\mathrm{z}$ axes respectively, $\mathrm{v}-$ is the initial velocity of the pulse.

So, based on the results of this paper, we can make the conclusion: in a photonic crystal with a spatially variable refractive index of carbon nanotubes, a stable propagation of a two-dimensional FCOP is possible. The dependences of the propagation of a twodimensional FCOP on the lattice constant and modulation depth have been also determined. When these parameters change, the propagation velocity and the shape of the pulse also change. Thus, changing the parameters of the medium, we can control the properties of the pulse, which is important for practical applications.

The reported study was funded by RFBR and the government of Volgograd region according to the research project 19-42-343002.

\section{References}

1. G. Fibich, B. Ilan, Opt. Let., 29, 8 (2004).

2. A. Mekis, J.C. Chen, I. Kurland, S. Fan, P.R. Villeneuve, J.D. Joannopoulos, Phys. Rev. Lett., 77, 3787 (1996).

3. A.V. Zhukov, R. Bouffanais, M.B. Belonenko, I.S. Dvuzhilov, Yu.V. Nevzorova, Appl. Phys. B., 123, 196 (2017). 